\title{
A hydrodynamical model for the Fermi-LAT $\gamma$-ray light curve of blazar PKS 1510-089
}

\author{
J.I. Cabrera ${ }^{1, a}$, Y. Coronado ${ }^{1, b}$, E. Benítez ${ }^{1, c}$, S. Mendoza ${ }^{1, d}$, D. Hiriart ${ }^{2, e}$, and M. Sorcia ${ }^{1, f}$ \\ ${ }^{1}$ Instituto de Astronomía, Universidad Nacional Autónoma de México, AP 70-264, 004510 Distrito Federal, México \\ ${ }^{2}$ Instituto de Astronomía, Universidad Nacional Autónoma de México, AP 877, 22800 Ensenada, BC, México.
}

\begin{abstract}
A physical description of the formation and propagation of working surfaces inside the relativistic jet of the blazar PKS 1510-089 are used to model its $\gamma$-ray variability light curve using Fermi-LAT data from 2008 to 2012. The physical model is based on conservation laws of mass and momentum at the working surface as explained by Mendoza et al. (2009). The hydrodynamical description of a working surface is parametrized by the initial velocity and mass injection rate at the base of the jet. We show that periodic variations on the injected velocity profiles are able to account for the observed luminosity, fixing model parameters such as mass ejection rates of the central engine injected at the base of the jet, oscillation frequencies of the flow and maximum Lorentz factors of the bulk flow during a particular burst.
\end{abstract}

\section{Introduction}

Among all types of AGN, Blazars (Blazar class is defined as radio loud sources conformed by the BL Lac objects and the flat-spectrum radio quasars -FSRQ, represent the most energetic class. They are known to have the most powerful jets and also show a highly variable spectral energy distribution (SED) from the radio to the $\gamma$-rays wavelengths.

The FSRQ PKS 1510-089 is known to be one of the most powerful astrophysical objects with a highly collimated relativistic jet that has shown apparent superluminal velocities between $20 c$ to $46 c$ and with a semi-angle aperture for the jet $\sim 0.2^{\circ}$. Since the angle between the relativistic jet and the observer's line of sight $\sim 1.4^{\circ}-$ $3^{\circ}$, the jet almost coincides with the observer's line of sight. PKS 1510-089 was one of the $\gamma$-ray sources detected by EGRET. It has been monitored at high energies with AGILE and by FERMI-LAT.It has also been studied with Major Atmospheric Gamma-ray Imaging Cherenkov Telescope (MAGIC) and High Energy Stereoscopic System (HESS). The most prominent outbursts displayed by PKS 1510-089 were reported in early 2009, 2010, 2012 and middle of 2011. The high activity observed in this source, turns it into an ideal target for the physical study of its highly relativistic jet.

Precise models for the light curve (LC) produced by the outburst and flares from blazars are not done using directly the data variations observed in different wave-

\footnotetext{
a e-mail: jcabrera@ciencias.unam.mx

be-mail: coronado@astro.unam.mx

ce-mail: erika@astro.unam.mx

de-mail: sergio@astro.unam.mx

e-mail: hiriart@astrosen.unam.mx

fe-mail: msorcia@astro.unam.mx
}

lengths. Instead, models are applied to explain the behaviour of the SED [e.g. 1, 2]. Direct understanding of the LC requires a precise knowledge of the hydrodynamical behaviour of the relativistic flow. Mendoza et. al. 2009, hereafter [3], have constructed a hydrodynamical model of the motion of a working surface inside a relativistic jet which is able to fit the observed LCs of long Gamma-Ray Bursts (1GRB's). Since the jets in Blazars are highly relativistic and their jet is nearly pointing towards the observer, similar to the jets observed in lGRB's, the physical ingredients of both phenomena can be considered the same but occurring at different physical scales of energy, sizes, masses, accretion rates, etc. [cf. 4].

The Blazar PKS 1510-089 is of tremendous importance since it exhibits extreme relativistic motions. As such, its energy curve must present luminosity variations and periods of extreme activity displayed as outbursts that, when physically modelled, can yield a better understanding of the physical parameters associated to the mechanism producing the observed luminosity.

We assume that the mechanism producing the observed LC in a typical IGRB is exactly the same that produces the variable LC of the Blazar PKS 1510-089. We thus apply the hydrodynamical jet model presented in [3] to the LC variations displayed by the Blazar PKS 1510089 in the $\gamma$-ray domain, using public data obtained with the FERMI-LAT telescope.

\section{Fermi-LAT Data}

The gamma-ray fluxes were obtained in the range of 0.2 to $300 \mathrm{GeV}$ using the public database of FERMI-LAT from 2008 August 08 to 2012 May 28. The $\gamma$-ray LC was constructed modelling the flux with a power law of the form 
$\mathrm{d} N / \mathrm{d} E=N_{0}\left(E / E_{0}\right)^{\gamma}$, with $\gamma=2-3$. The fluxes and errors obtained with the FERMI science tool package are given in photons $\times \mathrm{cm}^{-2} \mathrm{~s}^{-1}$. For further physical interpretation of the data, we have converted these fluxes and errors to $\mathrm{MeV} \mathrm{cm} \mathrm{cm}^{-2}$.

The photons considered for analysis were taken from a region centred on the coordinates of PKS 1510-089 with a radius of $15^{\circ}$. Figure 1 shows the $\gamma$-ray LC, with a bin size of 1 day. We chose these bins, since the errors are larger using shorter bin sizes, complicating the analysis of the data and because particular outbursts can be adequately resolved.

From Figure 1 it follows that the source displayed the historical maximum outburst in MJD 55851, corresponding to 2011 October 17. Another important outburst occurred in MJD 54899 (2009 March 9) and was observed with AGILE [5]. Note that [6] reports extra flares $<200 \mathrm{MeV}$ during the period $54850-54950 \mathrm{MJD}$, which are not seen in our $>200 \mathrm{MeV}$ selection.

\section{A hydrodynamical model for the Light Curve of PKS 1510-089}

The formation of internal shock waves on a relativistic jet are commonly explained by different mechanisms, such as the interaction of the jet with inhomogeneities of the surrounding medium, the bending of jets and time fluctuations in the parameters of the ejection [see e.g. 3, 7]. In particular, the model by [3] is a hydrodynamical description that can be applied to shock waves inside relativistic jets. This semi-analytical model describes the formation of a working surface inside a hydrodynamical jet due to periodic variations of the injected flow. When fast flow overtakes slow flow, an initial discontinuity is formed and a working surface is produced. The working surface travels along the jet and radiates away kinetic energy. The article by [3] assumed that the efficiency converting factor is $\sim 1$ and that it is mostly emitted in the $\gamma$-ray band. Following [3], we assume that flow is injected at the base of the jet with a periodic velocity given by

$$
v(\tau)=v_{0}+c \eta^{2} \sin \omega \tau,
$$

where $\tau$ is the time in the rest frame of the source, the velocity $v_{0}$ is the "background" bulk velocity of the flow inside the jet, and $\omega$ is the oscillation frequency. The positive constant parameter $\eta^{2}$ is chosen in such a way that oscillations of the flow are small so that the bulk velocity $v(\tau)$ of the flow does not exceed the velocity of light $c$. The mass ejection rate $\dot{m}(\tau)$ from the central engine which is injected at the base of the jet is assumed constant through a particular outburst event, but is allowed to vary from one outburst to another. The radiated energy of the flow as a function of time is calculated as the difference between the total energy $E_{0}$ injected at the base of the jet and the kinetic energy inside the working surface $E_{\mathrm{ws}}$. The luminosity $L$ is thus calculated as the derivative of this radiated energy with respect to time. As described by [3], there are two ways of calculating this luminosity curve. The first method consisted in a semi-analytical procedure and the second is performed with a full hydrodynamical numerical model. The authors showed that the semi-analytical model is in good agreement with the full numerical simulation, and as such we model the LC of PKS 1510-089 using their semi-analytical approach.

The semi-analytical approach is based on the assumption that equation (1) is valid and as such, one needs to know (or find through fits to observational data) the values of $v_{0}, \eta^{2}, \omega$ and $\dot{m}$. Furthermore, the mass ejection rate $\dot{m}$ enters in the description of the problem through the luminosity relation: $L \propto \dot{m} c^{2}$. The average bulk velocity $v_{0}$ must come from observational data, for this particular source[8] reports a value $\Gamma\left(v_{0}\right)=18$. With this, the model is left with three free parameters: $\eta^{2}, \dot{m}$, and $\omega$, which can be fixed by fitting the best theoretical LC to the observational data.

\section{Modelling the $\gamma$-ray Light Curve}

To model the LC of Figure 1, we have selected the most conspicuous flares. The criterion used consists of selecting only those flares that are beyond $3 \sigma$ noise level according to the errors shown in the LC. By doing so, it turns out that 38 relevant peaks were chosen for our fitting.

As explained in section 3 , the model has four free parameters. The velocity parameter $v_{0}$ for this particular object is such that its Lorentz factor is $\Gamma\left(v_{0}\right)=$ 18. To calculate the measured luminosity $L$ from the observed flux $F$, we multiply the observed flux $F$ by $[9,10]: 4 \pi D_{L}^{2} \delta^{-p}$ where the relativistic beaming $\delta:=$ $1 / \Gamma\left(v_{0}\right)\left(1-\left(v_{0} / c\right) \cos \theta\right) \sim 18$, for a luminosity distance $D_{L}$, which for this particular case is $D_{L}=1919 \mathrm{Mpc}$ and the angle $\theta \sim 1.4^{\circ}-3^{\circ}$ is the angle between the jet and the observer's line of sight (cf. Section 1). We have selected a beaming index $p=3$ in accordance with the results of [11] for Blazars and lGRB's.

The model presented by [3] is such that the theoretical luminosity and time are presented in a very particular system of units. To fit the best theoretical LC to the data, one needs to have a common system of units. To achieve this, we have normalised the "measured" Luminosity to its peak and the measured time to the FWHM of the measured LC. In order to compare with the theoretical model, the theoretical LC is also normalised to its peak and the time is normalised to the FWHM of the theoretical luminosity curve. Once both theoretical and measured LCs are in this common dimensionless system of units, this procedure allows us to fit the best theoretical LC by performing a $\chi^{2}$ statistical test to find the optimal parameter $\eta^{2}$. Note that in this normalised system of units, the model only depends on one free parameter: $\eta$. Once the value of $\eta$ is found, we can rescale back to physical units and in such a rescaling the parameters $\dot{m}$ and $\omega$ are obtained, since according to [3], $L \propto \dot{m} c^{2}$ and $t \propto \omega^{-1}$. The luminosity fits are then transformed to the observed flux dividing them by $4 \pi D_{L} / \delta^{3+\alpha}$. The results of these fits are shown in Figure 2 . The obtained values of the physical parameters of the model for each particular modelled outburst are presented in Table 1. 


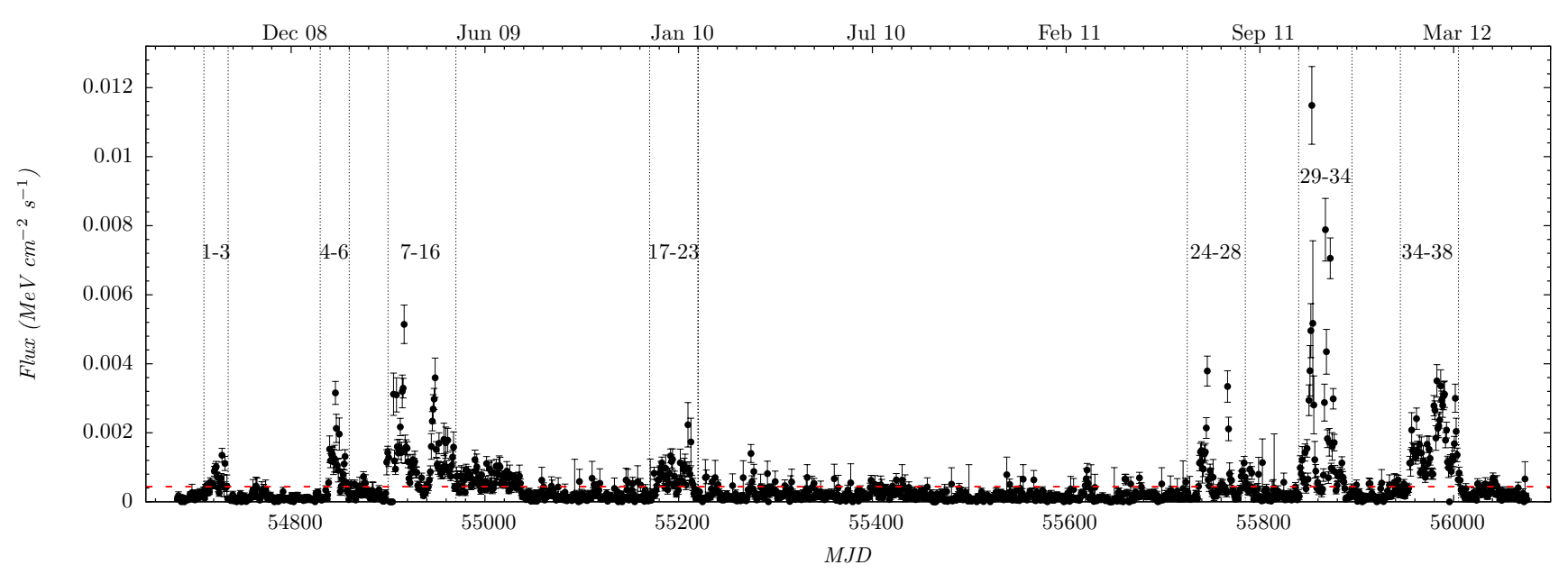

Figure 1. Fermi-LAT light curve of Blazar PKS 1510-089 (from 0.2 to $300 \mathrm{GeV}$ ) obtained from 2008 August to 2012 May. The outburst identification number (ID) labelled in the figure stands for the different flares selected in our work (see text). The $3 \sigma$ noise level is represented by the red horizontal dashed line.

There are a certain subclass of outbursts that we do not model. These outbursts, labelled 8, 10, 20, 27 and 32 in Figure 1, do not have enough data to allow us an accurate modelling. The outburst labelled 11 seems to have a fall that develops into a constant value before reaching an expected minimum and no data points further, so it seems incomplete. Outburst 14 has huge errors and the $\chi^{2}$ statistical test does not converge. Outbursts 34 and 35 have large errors which also makes the modelling not accurate.

\section{Discussion}

We have modelled the LC of Blazar PKS 1510-089 for almost 4 years using the hydrodynamical model of [3]. The modelling was performed by assuming a periodic velocity injection mechanism at the base of the relativistic jet that leads to the formation of a working surface and is capable of loosing energy as it travels along the jet. As explained in section 3, the model by [3] was constructed to deal with LCs of lGRB. However, the Blazar PKS 1510089 has many physical characteristics to be considered a geometrical large scaled version of a lGRB since it has a highly relativistic jet that points towards the observer. The results presented in Table 1 show high upper limits for the bulk Lorentz factors achieved with oscillations of the flow, that reach values as large as $\lesssim 380$ for one particular event. These inferred huge Lorentz factors in the bulk velocity oscillation of this Blazar show another close similarity with lGRB's.

The range of parameters as presented in Table 1, i.e. $\dot{m} \sim(2-25) \times 10^{-3} \mathrm{M}_{\odot} \mathrm{yr}^{-1}, \omega^{-1} \sim(0.3-2.6) \times 10^{3} \mathrm{~s}$ and variations of the Lorentz factor $\Gamma \sim 10-380$, denote a scaling between the lGRB counterparts found in [3] for which $\dot{m} \sim 10^{-1}-10^{-2} \mathrm{M}_{\odot} \mathrm{s}^{-1}, \omega^{-1} \sim 10 \mathrm{~s}$ and $\Gamma \sim 50-$ 500. Note that the maximum and minimum values of the Lorentz factor for a particular outburst take into account the observational errors of the LC. The real value lies in between those calculated ranges. In this particular case to the extreme relativistic motion of the jet in the Blazar PKS 1510-089. The energy released in each outburst can be calculated by taking the integral of the luminosity with respect to time, which occurs typically over periods of a few days. The value of this released energy is $\sim 10^{39}-$ $10^{40} \mathrm{~J}$, which shows the tremendous energy released by each individual outburst. This energy is to be compared with the energy released in about $10 \mathrm{~s}$ by a IGRB which is $\sim 10^{44} \mathrm{~J}$.

The most energetic burst, labelled 30, inyected at the base of the jet a total mass $m=\dot{m} \Delta t \sim 10^{-3} M_{\odot}$ while the burst lasted $\Delta t \sim 15$ days. Analysis of all bursts shows that the ejected mass interval is $10^{-5} M_{\odot} \lesssim m \lesssim 10^{-3} M_{\odot}$, for a time duration range 4 days $\lesssim \Delta t \lesssim 30$ days.

The variations of the injected flow at the base of the jet cause the formation of working surfaces that produce bursts of $\gamma$-rays in the structure of the jet. The physical mechanism producing the oscillations of the input flow, which allows fast fluid to overtake the slow one, leading to the formation of working surfaces, is beyond the scope of this work. the accretion-ejection mechanism associated to a particular object is not necessarily expected to be of constant velocity and mass accretion-ejection rates.

It is important to note that the assumption of seeing a Blazar as a scaled version of a lGRB is not new. In an early attempt for finding a unified model of jet and centralengine power, [4] made this identification. The more relativistic a Blazar jet is, the more it will resemble a lGRB. The idea of having a unified physical model for all types of astrophysical jets was first suggested by the pioneering works for the astrophysical scaling laws of black holes. The work presented strengths arguments about a unified picture of all astrophysical relativistic jets.

PKS 1510-089 resulted to be an ideal target to test the model by [3] since it closely resembles a lGRB in some of its outbursts. Future tests of the model have to be done with a wide variety of Light Curves from a large collection of Blazars and micro-quasars. 

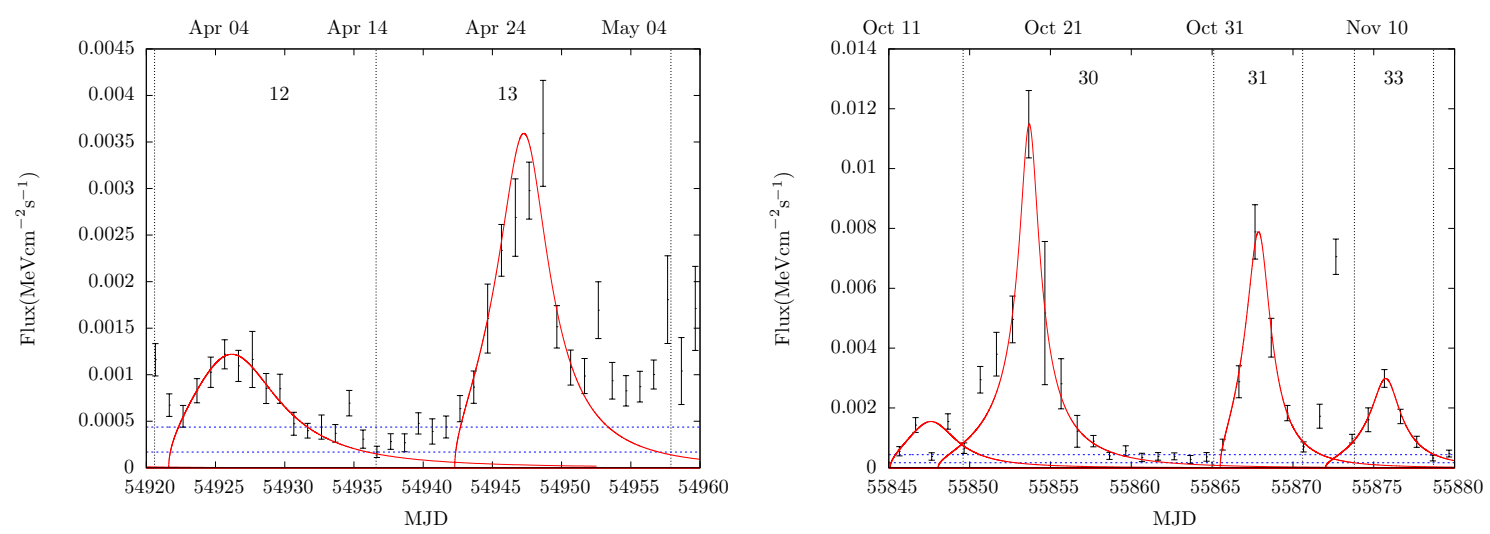

Figure 2. In each panel, the continuous red curve represents the fitting done to the light curve of PKS 1580-089 with the semi-analytical model of internal shock waves (working surfaces) by [3]. Blue horizontal dotted-lines in all panels show the $1 \sigma$ and $3 \sigma$ noise levels. The left panel shows a zoom of the peaks 12 and 13. The right panel shows a zoom of the prominent October 2011 outburst. This outburst is $\sim$ three times more luminous than the one observed in 2009 March. Up to now, this is the most violent outburst observed in the $\gamma$-ray waveband by FERMI.

Table 1. Different physical quantities obtained for the outbursts modelled in this work. The background Lorentz factor of the bulk velocity of the flow was assumed to be 18 . The first three columns from left to right are the date, numeric identification of the outburst (ID \#) and the date corresponding to the maximum luminosity for a particular outburst. Columns four and six are the obtained values for the parameters $\eta^{2}$ (measured in units of the speed of light $c$ ) and the inverse frequency $\omega^{-1}$ relevant to the particular variational model of equation (1). Column five corresponds to the maximum upper limit of the Lorentz factor of the flow for each particular outburst. The minimum Lorentz factor of the flow for all outbursts is $\sim 12-13$. Column seven represents the mass injection rate $\dot{m}$ of the flow at the base of the jet. The values of all inferred parameters are accurate to within $10 \%$.

\begin{tabular}{|c|c|c|c|c|c|c|}
\hline Date & $\begin{array}{r}\text { ID } \\
\#\end{array}$ & $\begin{array}{r}\text { MJD } \\
+54000\end{array}$ & $\begin{array}{l}\eta^{2} / c \\
10^{-3}\end{array}$ & $\Gamma_{\max }$ & $\begin{array}{c}\omega^{-1} \\
10^{3} \mathrm{~s}\end{array}$ & $\begin{array}{c}\dot{m} \\
10^{-3} \mathrm{M}_{\odot} / \mathrm{yr}\end{array}$ \\
\hline$\overline{08 \text { Sep }}$ & 1 & 722.66 & 1.500 & 106 & 1.05 & 2.16 \\
\hline 08 Sep & 2 & 728.66 & 1.520 & 143 & 0.50 & 2.87 \\
\hline 08 Sep & 3 & 731.66 & 1.510 & 120 & 0.41 & 2.37 \\
\hline 09 Jan & 5 & 849.66 & 1.501 & 107 & 0.34 & 4.18 \\
\hline 09 Jan & 6 & 855.66 & 1.533 & 209 & 1.49 & 2.80 \\
\hline 09 Mar & 7 & 899.66 & 1.330 & 48 & 0.94 & 3.04 \\
\hline 09 Mar & 9 & 908.66 & 1.460 & 76 & 0.37 & 6.61 \\
\hline 09 Apr & 12 & 925.66 & 1.430 & 66 & 1.27 & 2.60 \\
\hline $09 \mathrm{Apr}$ & 13 & 948.66 & 1.515 & 130 & 1.22 & 7.67 \\
\hline 09 May & 15 & 957.66 & 1.300 & 45 & 0.88 & 3.85 \\
\hline 09 May & 16 & 967.66 & 1.523 & 152 & 1.05 & 3.38 \\
\hline 09 Dic & 17 & 1182.66 & 1.534 & 219 & 2.60 & 2.40 \\
\hline 09 Dic & 18 & 1186.66 & 1.400 & 58 & 0.39 & 2.06 \\
\hline 09 Dic & 19 & 1191.66 & 1.488 & 94 & 1.24 & 2.84 \\
\hline $10 \mathrm{Jan}$ & 21 & 1205.66 & 1.510 & 120 & 1.04 & 2.23 \\
\hline $10 \mathrm{Jan}$ & 22 & 1209.66 & 1.493 & 98 & 0.95 & 4.76 \\
\hline $10 \mathrm{Mar}$ & 23 & 1274.66 & 1.430 & 66 & 0.68 & 2.99 \\
\hline 11 Jun & 24 & 1739.66 & 1.460 & 76 & 0.74 & 3.16 \\
\hline $11 \mathrm{Jul}$ & 25 & 1745.66 & 1.527 & 169 & 0.81 & 8.09 \\
\hline $11 \mathrm{Jul}$ & 26 & 1766.66 & 1.469 & 81 & 0.36 & 7.13 \\
\hline 11 Aug & 28 & 1783.66 & 1.380 & 55 & 0.41 & 2.40 \\
\hline $11 \mathrm{Oct}$ & 29 & 1848.66 & 1.460 & 76 & 0.67 & 3.30 \\
\hline $11 \mathrm{Oct}$ & 30 & 1853.66 & 1.541 & 383 & 1.32 & 24.52 \\
\hline $11 \mathrm{Nov}$ & 31 & 1867.66 & 1.522 & 149 & 0.57 & 16.83 \\
\hline $11 \mathrm{Nov}$ & 33 & 1875.66 & 1.531 & 193 & 0.88 & 6.37 \\
\hline $12 \mathrm{Feb}$ & 36 & 1972.66 & 1.220 & 39 & 0.66 & 3.55 \\
\hline $12 \mathrm{Mar}$ & 37 & 1982.66 & 1.350 & 50 & 2.03 & 7.48 \\
\hline
\end{tabular}

\section{ACKNOWLEDGMENTS}

We thank the anonymous referee for his valuable comments who helped us to produce a much improved version of our letter. This work was supported by three DGAPA-UNAM grants (PAPIIT IN116210-3, IN1162113, IN111513-3). JIC acknowledges support given by IAUNAM as a visiting researcher. JIC, YUC, EB, SM, DH and MS thank support granted by CONACyT: 50102, 210965, 13654, 26344, 8366, 177304. The authors acknowledge the use of the FERMI-LAT publicly available data as well as the public data reduction software.

\section{References}

[1] A.A. Abdo, M. Ackermann, I. Agudo, M. Ajello, H.D. Aller, M.F. Aller, E. Angelakis, A.A. Arkharov, M. Axelsson, U. Bach et al., ApJ716, 30 (2010), 0912.2040

[2] F. D'Ammando et al., Astronomy and Astrophysics529, A145 (2011), 1103. 3647

[3] S. Mendoza, J.C. Hidalgo, D. Olvera, J.I. Cabrera, MNRAS395, 1403 (2009), 0710 . 3806

[4] I.F. Mirabel, L.F. Rodriguez, Sky and Telescope103, 050000 (2002)

[5] F. D'Ammando et al., Astronomy and Astrophysics508, 181 (2009), 0909. 3484

[6] A.P. Marscher et al., ApJL710, L126 (2010), 1001.2574

[7] M.J. Rees, P. Meszaros, ApJL430, L93 (1994), arXiv: astro-ph/9404038

[8] F. D'Ammando et al., The Astronomer's Telegram 1436, 1 (2008)

[9] C.D. Dermer, G. Menon, High Energy Radiation from Black Holes: Gamma Rays, Cosmic Rays, and Neutrinos (2009)

[10] M.S. Longair, High Energy Astrophysics (2011)

[11] Q. Wu, Y.C. Zou, X. Cao, D.X. Wang, L. Chen, ApJL740, L21 (2011), 1108. 1442 